www.jmscr.igmpublication.org

Index Copernicus Value: 79.54

ISSN (e)-2347-176x ISSN (p) 2455-0450

crossrefDOI: https://dx.doi.org/10.18535/jmscr/v7i3.39

Journal Of Medical Science And Clinical Research

IGM Publication

An official Publication of IGM Publication

\title{
Is plasma free metanephrines accurate in the diagnosis of pheochrmocytoma in adults?
}

Authors

\author{
Marwan Said Muhanna Al-Riyami ${ }^{1}$, Dr Hassan Sadek ${ }^{2}$ \\ ${ }^{1}$ Chemical Pathology Laboratory, Royal Hospital, Ministry of Health Oman \\ ${ }^{2}$ Senior Consultant Lecturer, Oman College of Heath Sciences, Ministry of Health, Oman
}

\begin{abstract}
Background: Pheochromocytoma is a rare adrenaline-producing tumour that arises from adrenal gland. The number of cases are increasing globally each year. Pheochrmocytomas release a hormone called adrenaline which can cause severe health problems such as hypertension (high blood pressure), heart attack, and stroke. They might lead to death if not diagnosed early. Diagnosis of pheochromocytomas can be performed by different biochemical tests like catecholamines, metanephrines, chromogranin A ...etc.

Objective: To determine the efficacy of plasma free metanephrines measurements for inclusion and confirmation of pheochromocytoma.

Methods: Multiple electronic searches were carried out to determine studies in the English-language. Databases used for this purpose were CINHAL, AMED, MEDLINE, PubMed, ScienceDirect, Google scholar and the Cochrane library. To identify relevant articles, various key terms were used. The articles obtained were published during or after 2005. Moreover, inclusion and exclusion criteria were applied while performing this search. The intervention was plasma free metanephrines and comparison with other biochemical test such as plasma or urinary catecholamines, urinary metanephrines, chromogranin A and deconjugated plasma metanephrines.

Results: From forty-four studies, only seven studies corresponded with inclusion and exclusion criteria. The main results of four studies showed that plasma free metanephrines were highly efficient in the diagnosis of pheochrmocytoma compared to plasma catecholamines, epinephrines, urine metanephrines and plasma deconjugated metanephrines. The remaining three studies were displayed totally different findings. Urinary metanephrine, chromogranin A and urinary catecholamines demonstrated high sensitivities and specificities compared to plasma free metanephrines. Certain procedures like fast, abstinence from drugs; supine position should be followed by participants before and during blood and urine collection.

Conclusion: Plasma free metanephrines measurements are preferable (superior) in the diagnosis of pheochromocytoma. However, patients need to fast, abstinence from drugs, avoid caffeinated and decaffeinated beverages and blood should be collected in the supine position.
\end{abstract}

\section{Introduction}

Pheochromocytoma is a rare adrenaline-producing tumor that arises from adrenal gland. Each year about 1,000 new cases are identified in the United
States. It causes less than $0.2 \%$ of cases of high blood pressure (hypertension). The growth of pheochromocytomas takes place within the center portion of the adrenal gland called adrenal 
medulla which is an area in the peripheral nervous system (the nerves outside the brain and spinal cord). The main function of the adrenal medulla is the production of adrenaline (also called epinephrine or catecholamine). Adrenaline is the hormone that influence some functions of the body such as breathing, sexual arousal, digestion, blood pressure and heart rate. Additionally, it maintains the body to be ready to respond to stressful circumstances. It is released normally from the adrenal medulla in case of any danger or emergency situation. A part from increasing heart rate and blood pressure adrenaline also keeps air passages open in order to allow in sufficient oxygen, and increases oxygen and glucose supply to the brain and muscles (AAES, 2014).

Pheochromocytomas release adrenaline in an uncontrolled amount and this can lead to dangerous health issues like heart attack, stroke and possibility of death. "Pharmacological time bomb" is the term used by some clinicians on account of its danger (AAES, 2014).

Around $95 \%$ of adrenal-secreting tumors are existed within the abdomen ( 85 to $90 \%$ within the adrenal gland). $10 \%$ of the pheochromocytomas are bilateral (within both adrenal glands), $10 \%$ are discovered in children, $10 \%$ are inherited (genetic), $10 \%$ are cancer and $10 \%$ are positioned outside the adrenal gland. For these reasons it described as " $10 \%$ tumors". The majority of pheochromocytomas happened spontaneously, so there is an opportunity to develop this condition at any age (from very young to the elderly) (AAES, 2014).

Pheochromocytomas are frequently considered in the differential diagnosis of severe or atypical hypertension and can lead to death if not diagnosed early (Algeciras-Schimnich et al, 2008).

The first recognition of clinically significant pheochromocytoma was in 1926 when Cesar Roux in Switzerland and Charles H (Kudva et al, 2003).

Pheochromocytomas lead to high blood pressure (caused by excessive adrenaline production) characterized by the following symptoms: headaches, sweating, pounding of the heart, being shaky and becoming extremely pale. In addition, pheochromocytoma could be a part of another case known as Multiple Endocrine Neoplasia syndrome (MEN) in which patients mostly suffering from another cancers and issues involving hormones (MedlinePlus, 2014).

The diagnosis of pheochromocytoma depends essentially on the indication of excessive production of catecholamines. However, this step is filled with difficulties specifically falsenegative test results. Furthermore, false-positive results are a common and upsetting occurrence, because of low prevalence of pheochromocytoma in the tested subjects and insufficient specificity of biochemical tests. These difficulties in biochemical identification demonstrate a necessity for more sensitive and specific test that can effectively exclude or confirm pheochromocytoma. Several studies were conducted in order to determine the diagnostic performance for these tests. Various sample size, sample types and comparison groups were used (Jacques et al, 2002).

Recently, some authors have reported supremacy of metanephrines more than catecholamines in the diagnosis of pheochromocytoma. Others recommended the estimation of plasma free metanephrines as the first test of choice due to their preferable sensitivity and specificity but there is still disagreement and debating about whether plasma free metanephrine investigation is appropriate as a first line test in excluding and confirming pheochromocytoma (Vaclavik et al, 2007).

The plasma free metanephrine is one of the blood tests commonly ordered in subject with signs and symptoms of pheochromocytoma or with family history of pheochromocytoma. This investigation assesses blood for metanephrines and normetanephrines (metabolized forms of epinephrine and norepinephrine). Metanephrines can be measured in blood and urine. Other laboratory tests such as plasma catecholamines, 


\section{JMSCR Vol||07||Issue||03||Page 213-221||March}

urine catecholamines, chromogranin $\mathrm{A}$ and deconjugated metanephrines are also ordered in patients with characteristics of pheochromocytoma but their accuracy in the diagnosis of pheochromocytoma varies from one to another. These biochemical screening tests are institution and laboratory-dependent with changeable efficiency. The idealistic test to exclude or confirm pheochromocytoma has been discussed and examined over the years because of false results.
In Oman, plasma metanephrines, urinary catecholamines and urinary metanephrines are mainly requested in ruling out pheochromocytoma. Aveyard (2007) model is used to build the literature review (appendix1). The aim of this critical review is to figure out the accuracy of free plasma metanephrine in the diagnosis of pheochromocytoma in adult population.

\section{Appendix (1): Model of literature review (Aveyard, 2007)}

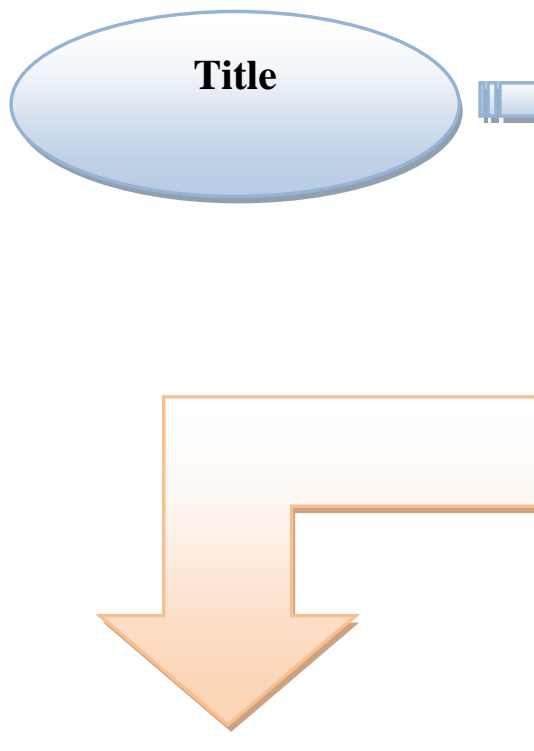

\section{Acknowledgment}
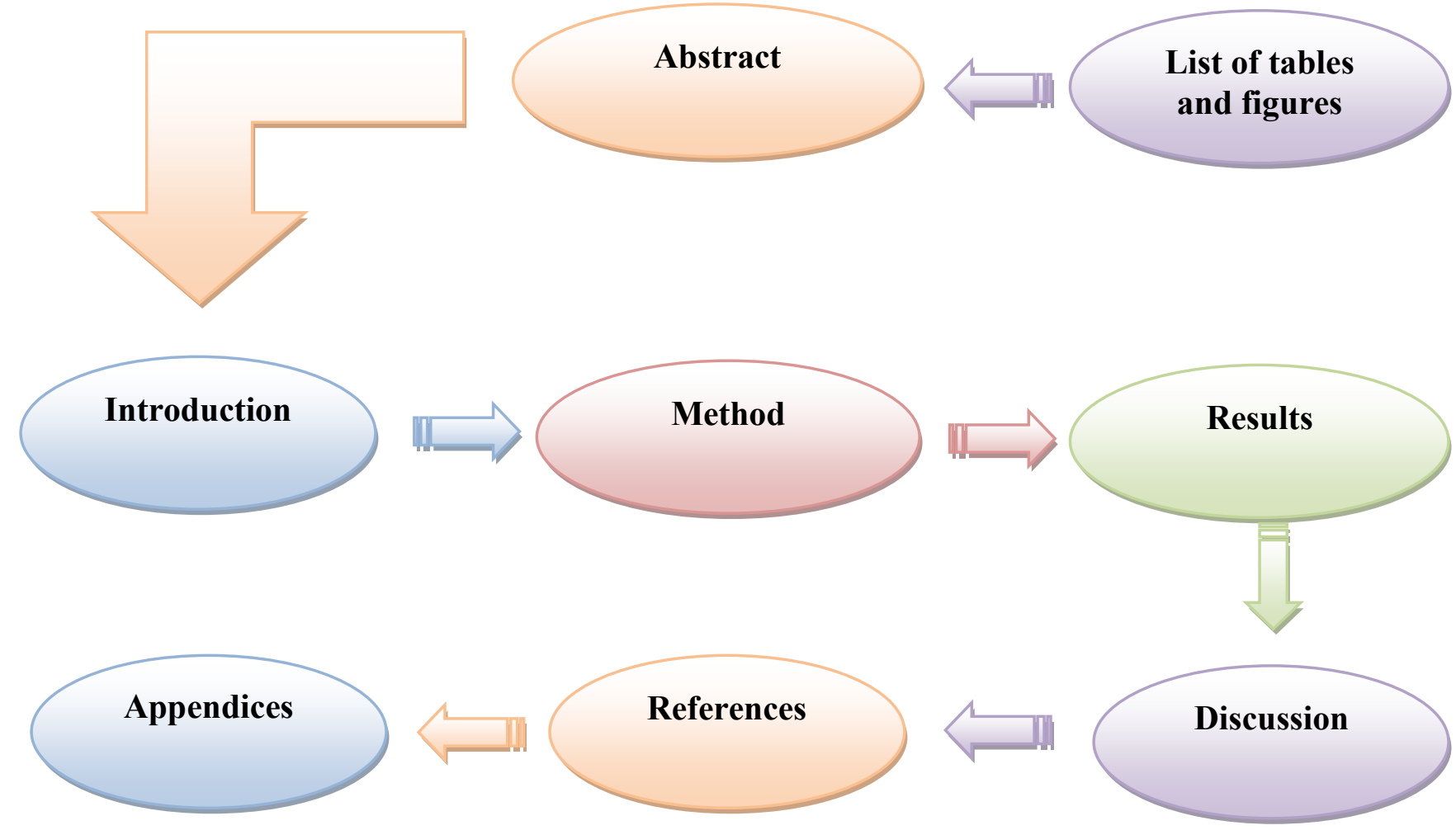

\section{Methods}

\section{Objective}

To review the suitable articles that provides evidences on the plasma free metanephrines in the diagnosis of pheochromocytoma in adult population.
Criteria taking into account for articles selection

* Types of study: the search mainly focused on randomized controlled trials (RCTs) but in this topic it was difficult to obtain RCTs. Other kinds of studies like 
prospective, cross-sectional, retrospectives and diagnostic studies were chosen as they were comparing the sensitivity and specificity of plasma free metanephrines with other methods concerning the same purpose (diagnosis of pheochromocytoma).

* Type of populations: adult patients (age $\geq$ 18 years )

* Type of Intervention: Plasma free metanephrine detection in order to diagnose adrenaline-producing tumor (pheochromocytoma)

* Type of outcome measure: the main outcome measured in the selected studies was to determine the sensitivity and specificity of plasma free metanephrines in excluding and confirming pheochromocytoma.

\section{Literature search}

\section{- Electronic Search}

Various electronic databases such as CINHAL, MEDLINE, AMED, PubMed, Science Direct and the Cochrane library were utilized to search for the relevant articles. Different key terms were used to find out the suitable studies which are associated to the topic. At the time of searching each word was used with its different spellings and synonyms in order to expand the search and maximum appropriate articles were obtained. The following keywords were used in the literature search: free plasma metanephrines, accuracy, diagnosis, evaluation, pheochromocytoma, adrenaline-producing tumors, catecholamine metabolites and investigation. The key terms were used according to PICO question. Additionally, the obtained relevant articles were read and checked in order to match the inclusion criteria of the literature search. At the end of this search 44 articles which were relevant to the topic.
The selected articles were published during the period from 2005 to 2014. Updated studies were considered while reviewing the articles and that was performed for the purpose of filtering the search on the studies which applying the international guidelines in the diagnosis patients with signs and symptoms of pheochromocytoma. Furthermore, the number of articles was limited by reading the abstract rather than reading the full text and wasting time for irrelevant articles. The abstract defined as a synopsis (summary) of the essay and generally provides an inclusive idea regarding the study.

Further, to choose the most appropriate studies and contribute it in the review as evidence, inclusion and exclusion criteria were proposed and taking into consideration.

\section{Inclusion Criteria}

* Adult subjects

* Measuring Free plasma metanephrines (sensitivity and specificity)

* Comparison with other method for the same purpose (diagnosis of pheochromocytoma)

* Conducted on human species.

* Both tests (intervention and comparator) were performed in the same period (Not the intervention now and the comparator after one month)

\section{Exclusion Criteria}

* Performed on pediatrics

* Performed on animals

* Plasma free metanephrines not a part of the study

* Non-English articles

* The purpose of the study was not detection of pheochromocytoma (e.g. medicationrelated influences)

* No comparator (plasma free metanephrines alone) 
* Conducted for the evaluation of certain analyzer or to create a reference range for metanephrine test

* Both the intervention and the comparator were not biochemical (One was biochemical while other was radiological).

\section{Review methods}

* Collection and analysis of data: Each article was evaluated separately. Details from each article were compared to identify if they are identical. The main outcome was to verify the sensitivity and specificity of plasma free metanephrines and other parameters (like catecholamines, deconjugated plasma metanephrines, etc) in the identification of pheochromocytoma.

* Appraisal tool: Each study was evaluated using studies of diagnostic accuracy methodology checklist according to Scottish Intercollegiate Guidelines Network (SIGN, 2014).

\section{Results}

\section{Studies included in the critical review:}

Approximately 44 articles were identified from different databases mentioned earlier. Of these 44 studies, 22 were removed because of duplication. However, 22 articles were screened, 6 were excluded as they did not match the selection criteria. 8 of the remaining articles were excluded because of population features (paediatrics) and 7 studies were corresponded with inclusion criteria (appendix 4, PRISMA Flow Diagram). The appropriate studies were authored by (Vaclavik et al, 2007; Herbomez et al, 2007; Hickman et al, 2009; Procopiou et al, 2009; Grouzmann et al, 2010 and Christensen et al, 2011).

The $7^{\text {th }}$ selected studies were published in English language during the period from 2005 to 2014. They were diagnostic, prospective, crosssectional, and retrospective. The appraisal tool used to evaluate these relevant articles was SIGN for studies of diagnostic accuracy. All studies were conducted with a view to assess the diagnostic capability of plasma free metanephrines in the detection of pheochromocytoma. Plasma free metanephrine test was compared with other biochemical markers like blood normetanephrines, blood plasma deconjugated metanephrines, blood (serum) chromogranin A, blood catecholamines and Urine epinephrines (catecholamines).

\section{Patient characteristics}

The populations included in these studies were adults (age $>18$ years) from both sexes. Some of them were previously or currently with family histories and suspected cases of pheochromocytoma while others were normal (they considered as controls). Pheochromocytoma was confirmed in some of these subjects and excluded in others. The sample size varies from study to another; the smallest number was 93 (Hickman et al, 2009) while the largest one was 1260 (Vaclavik et al, 2007).

\section{Biochemical markers}

Blood (venous) and urine (24 hours collection) samples were collected from each individual and were analyzed for the intervention (plasma free metanephrine) and the comparator (other parameters mentioned above). Blood samples were collected from patients lying supine (Vaclavik et al, 2007 and Pamporaki et al, 2013) while others in sitting position (Christensen et al, 2011). The remaining studies (Herbomez et al, 2007; Hickman et al, 2009 and Procopiou et al, 2009) did not mention the type of patient's position while blood was collected. The following table demonstrates the requirement (from each participant) prior blood sample collection: 
Table (1): Patients status prior samples collection

\begin{tabular}{|l|c|c|c|c|}
\hline Study & Fasting & Drugs arrested & $\begin{array}{c}\text { Avoid caffeinated } \\
\text { beverages }\end{array}$ & Others \\
\hline Vaclavik et al $\mathbf{( 2 0 0 7 )}$ & $\begin{array}{c}\text { Not } \\
\text { mentioned }\end{array}$ & $\begin{array}{c}\text { Yes except } \\
\text { paracetamol }\end{array}$ & Yes & Low-risk population \\
\hline Herbomez et al (2007) & Yes & Not all & Not mentioned & Use of Omeprazole \\
\hline Hickman et al (2009) & Yes & Yes & Yes & - \\
\hline Procopiou et al $\mathbf{( 2 0 0 9}$ & Yes & Yes & Yes & - \\
\hline Grouzmann et al (2010) & Yes & Yes & Yes & - \\
\hline Christensen et al (2011) & No & Not clearly given & Yes & $\begin{array}{c}\text { Blood collected in } \\
\text { sitting position }\end{array}$ \\
\hline Pamporaki et al (2013) & Yes & Yes & Yes & - \\
\hline
\end{tabular}

\section{Diagnostic performance}

Statistical analysis supported that plasma free metanephrines were more sensitive and specific in the diagnosis of pheochromocytoma than other biochemical markers. The sensitivity and specificity were based on 95\% CI reference range. In one study (Vaclavik et al, 2007) plasma normetanephrines were more sensitive (96\%) and specific $(98.7 \%)$ than plasma free metanephrines (sensitivity $88 \% \quad \& \quad$ specificity $95.1 \%$ ). Chromogranin A showed high sensitivity and specificity rates (98.3\% and $97 \%$, respectively) compared to plasma free metanephrines (sensitivity $71 \%$ \& specificity 89.9 ) in patients with renal insufficiency (Herbomez et al, 2007). Hickman et al (2009) mentioned that plasma free metanephrine was superior in sensitivity $(100 \%)$ and specificity $(97.6 \%)$ when tested in parallel with plasma catecholamines (sensitivity $78.6 \%$ \& specificity $70.7 \%$ ). Procopiou et al (2009) were displayed better diagnostic performance of plasma free metanephrine (sensitivity $91.7 \%$ \& specificity $100 \%$ ) than 24 hours urine epinephrine (sensitivity $83.3 \%$ \& specificity $90.1 \%$ ) but these findings were not supported by Christensen et al (2011) in case of sensitivity because the sensitivity $(100 \%)$ urine catecholamines was better than that of plasma free metanephrine $(91 \%)$. Plasma free metanephrine presented with effective sensitivity and specificity $(97 \% \&$ \& $93 \%$, respectively) compared to deconjugated plasma metanephrines (sensitivity $92 \%$ \& specificity $89 \%$ ) when assessed by Pamporaki et al (2013).

\section{Discussion}

The aim of this literature review was to disclose the diagnostic efficiency of plasma free metanephrines in comparison to other biochemical tests such normetanephrines, chromogranin A, urine catecholamines, plasma catecholamines, urine metanephrines, deconjugated plasma metanephrines and epinephrine (urine catecholamines) in the diagnosis of pheochromocytoma. Seven articles were included in this review. These selected articles were critically appraised for the measurements and the outcome. Each study was comparing plasma free metanephrine (intervention) with different test (comparator). To be more specific, Vaclavik et al (2007) were measured plasma free metanephrines with normetanephrines; Herbomez et al (2007) had tested plasma free metanephrine along with chromogranin A; Hickman et al (2009) had quantified plasma free metanephrines at the same time as plasma catecholamines. Different studies had investigated plasma free metanephrines in the company of urine metanephrines (Grouzmann et al, 2010) and deconjugated plasma metanephrines (Pamporaki et al, 2013). Also, plasma free metanephrines were examined together with urine catecholamines by Procopiou et al (2009) and Christensen et al (2011).

Sample size was differing in each study. The smallest number was 93 (Hickman et al, 2009) participants while the largest one was 1260 (Vaclavik et al, 2007). The remaining was ranged between 134 and 988. Moreover, the gender of participants involved in these studies was males and females. The age of subjects ranged between 
32 years and 59 years. 39.6 years in Hickman et al (2009) was the youngest mean age while Herbomez et al (2007) showed the oldest mean age of 59 years. Some of these studies were performed in multi-centres with multi nationality participants (Grouzmann et al, 2010 \& Vaclavik et $a l, 2007)$ while others were conducted at single centres with individuals of a single nationality (Christensen et al, 2011 \& Procopiou et al, 2009).Three studies were done as following: one study (Hickman et al, 2009) was carried out in single centre with multi-nationality people, one was implemented in multi-centres (Vaclavic et al, 2006); and the last one (Herbomez et al, 2007) in single centre (nationality was not mentioned in these two studies). All studies were approved by the institutional review boards.

The recruitment criteria of subjects were similar in some studies but differ in others. For instance, normotensive populations were enrolled as controls only in two studies (Hickman et al, 2007 \& Pamporaki et al, 2013). The majority of the involved articles tested patients with pheochromocytoma, hypertension, adrenal mass, familial history of pheochromocytoma and incidentaloma. Intensive care unit and hemodialysis patients were examined for pheochromocytoma in a single study (Hickman et al, 2009). Additionally, renal insufficiency patients were included by Herbomez et al (2007).

Blood specimens were collected from the whole participants using forearm intravenous cannula while 24 hours acidified urine was utilized in measuring urine metanephrines and urine catecholamines. Plasma free metanephrines were highly efficient in the diagnosis of pheochromocytoma in case of fasting, abstinence from paracetamol and stop caffeinated and decaffeinated beverages before blood collection and 24 hours urine collection. In addition, before and during blood collection, patient should relax in supine position in a noiseless room for approximately 20-30 minutes. Once sample was collected it should be kept on ice and stored at -80 ${ }^{0} \mathrm{C}$ till it analyzed.
In term of diagnostic performance, the findings of this review could be divided into two categories. First category includes three studies (Vaclavik et al (2007), Herbomez et al (2007) and Christensen et al (2011)). In this group the sensitivity and specificity of plasma free metanephrines were lower than those of comparators (urine metanephrine, chromogranin A and urine catecholamines). Specifically, sensitivity of free plasma metanephrines were ranged from $71 \%$ to $91 \%$ (95\% CI $68.8 \%$ - 99.2\%) while specificity extended from $89.9 \%$ to $98.3 \%$ (95\% CI $68.8 \%$ $99.2 \%$ ). Sensitivities of the other tests (mentioned earlier) were stretched between $96 \%$ and $100 \%$ (95\% CI 68.8\% - 100\%) whereas specificities were ranged from $94 \%$ to $98.7 \%$ (95\% CI $68.8 \%$ $99.2 \%)$. Several factors were involved in reducing the efficiency of plasma free metanephrines like conducting studies in low-risk populations and in cases where imaging was difficult to confirm the disease (pheochromocytoma) in all participants (Vaclavik et al, 2007). Other reasons were that drugs were partially arrested because some drugs like omeprazole (drug used to treat symptoms of gastroesophageal reflux disease (GERD) and help in the healing of erosive esophagitis (Drugs.com, 2014) could lead to elevate chromogranin A (Herbomez et al, 2007). Collection of sample from people who were not fasting, did not abstain from paracetamol in sitting position could reduce the accuracy of plasma free metanephrines (Christensen et al, 2011).

On the other hand, four studies were included in the second group (Hickman et al (2009), Procopiou et al (2009), Grouzmann et al (2010) and Pamporaki et al (2013)). They had displayed totally different findings. To be more specific, sensitivities and specificities of plasma free metanephrines were indicated highly efficient for the diagnosis of pheochromocytoma compared to other biochemical markers like plasma catecholamines, epinephrines, urine metanephrines and plasma deconjugated metanephrines. Testing results indicated sensitivities of 91.7 to $100 \%$ compared to $78.7 \%$ 
to $83.3 \%$ for plasma \& urinary catecholamines and $92 \%$ to $95 \%$ for urinary or plasma metanephrines. Recruitments of populations in these studies and procedures followed prior blood and 24 hours urine collection played very important role in making sensitivity and specificity of plasma free metanephrines highly effective in the identification of pheochromocytoma. For instance, enrolment of patient known with radiological and histological proven pheochromocytoma (Hickman et al, 2009), participants were fasting, abstained from paracetamol (as it cross react with assays), stop caffeinated and decaffeinated beverages, patients were rested for approximately 20-30 minutes (prior blood \& urine collections) and blood was collected in supine positions (Procopiou et al, 2009), Grouzmann et al, 2010) and Pamporaki et al, 2013). Furthermore, after blood drawn, samples were kept on ice; centrifuged at $4^{\circ} \mathrm{C}$ and stored at $-80{ }^{0} \mathrm{C}$ till analysis (see figure 1).

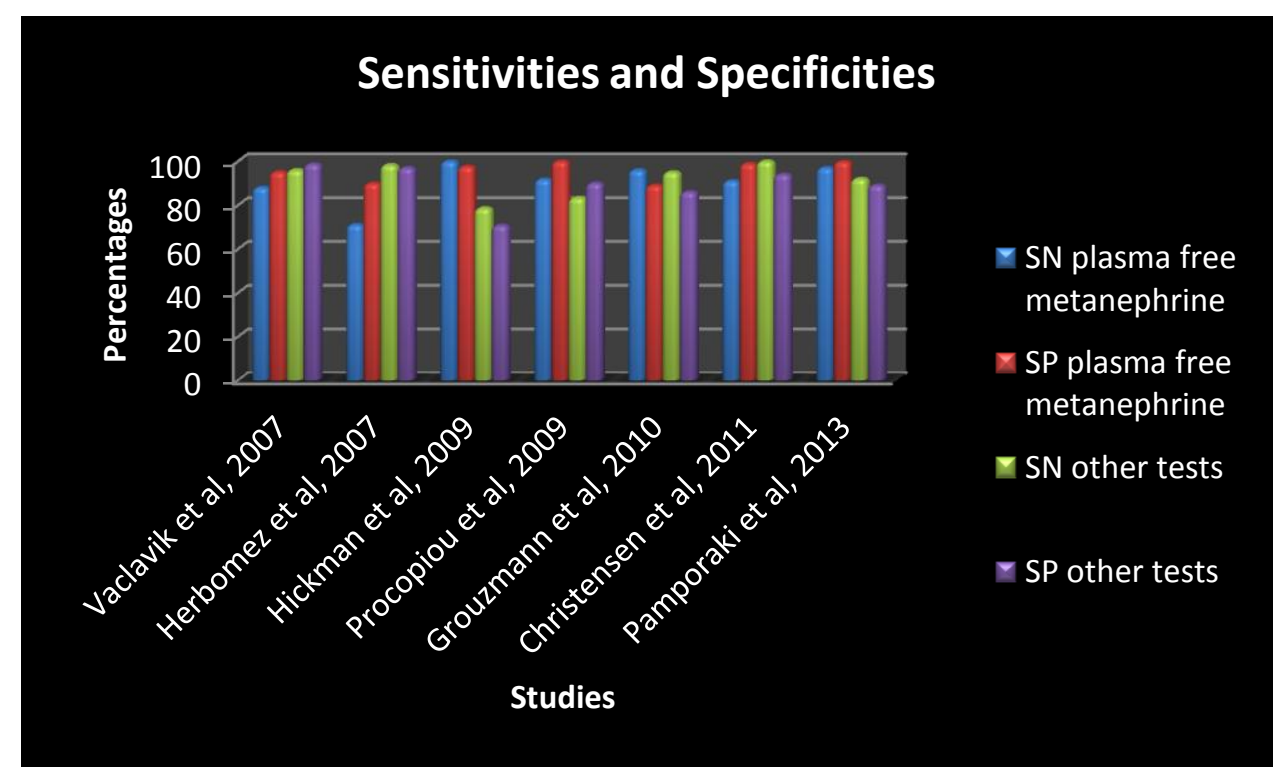

Figure 1: Sensitivities (SN) and specificities (SP) of plasma free metanephrines and other tests

In Oman, pheochromocytoma diagnosed only at SQUH using catecholamines and chromogranin A (Sanath et al, 2014) and the royal hospital using plasma/urinary metanephrines and urinary catecholamines.

\section{Limitations of this critical review}

Some limitations were noticed while undertaking this review. Studies included in this review were all published in English language so; important studies published in other languages regarding the same topic might be missed. Moreover, the majority of the selected articles were conducted in single centres with single nationality population and small sample size.

\section{Conclusion}

Plasma free metanephrines measurements are efficient in the diagnosis of pheochromocytoma. However, patients need to fast, abstinence from drugs, avoid caffeinated and decaffeinated beverages and blood should be collected in the supine position.

\section{References}

1. Aveyard, H. 2007, "A suggested structure" in: Doing a literature review in health and social care: a practical guide, Open University Press, Maidenhead. pp. 148- 151.

2. Christensen, T. T., Frystyk, J., \& Poulsen, P. L., "Comparison of plasma metanephrines measured by a commercial immunoassay and urinary catecholamines in the diagnosis of pheochromocytoma", Scandinavian 
Journal of Clinical \& Laboratory Investigation, no. 71, pp. 695-700.

3. Grouzmann, E., Drouard-Troalen, L., Baudin, E., Pierre-Francois, P., Muller, B., Daniela, G., et al. 2010, "Diagnostic accuracy of free and total metanephrines in plasma and fractionated metanephrines in urine of patients with pheochromocytoma", European Journal of Endocrinology, no. 162, pp. 951-960.

4. Herbomez, M., Forzy, G., Bauters, C., Tierny, C., Pigny, P., Carnaille, B., et al. 2007, "An analysis of the biochemical diagnosis of 66 pheochromocytoma", European Journal of Endocrinology, no. 156, pp. 569-575.

5. Hickman, P., Leong, M., Chang, J., Susan, R., \& McWhinney, B. 2009, "Plasma free metanephrines are superior to urine and plasma catecholamines and urine catecholamine metabolites for the investigation of pheochromocytoma", The Journal of Pathology, vol. 41, no. 2, pp. 173-177.

6. Jacques, W. M., Karel Pacak, McClellan, M., Marston Linehan, W., Massimo Mannelli, Peter Friberg, et al, 2002, "Biochemical Diagnosis of Pheochromocytoma, Which Test Is Best?", The Journal of the American Medical Association, vol. 287, no. 11, pp. 14271434.

7. Omeprazole, 2014, [online]. Available from: http://www.drugs.com/omeprazole.html [Accessed 25 March 2014].

8. Pamporaki, C., Darr, R., Bursztyn, M., Glockner, S., Bornstein, S. R., Lenders, J. W. M., et al. 2013, "Plasma-free vs deconjugated metanephrines for diagnosis of pheochromocytoma”, Clinical Endocrinology, no. 79, pp. 476-483.

9. Pheochromocytoma (adrenaline-producing adrenal tumor), 2014, [online]. Available from:

http://endocrinediseases.org/adrenal/pheochr omocytoma.shtml [Accessed 25 March 2014].

10. Pheochromocytoma and diabetes mellitus, 2014 [online]. Available from: http://www.diapedia.org/other-types-ofdiabetes-mellitus/phaeochromocytoma-anddiabetes-mellitus [Accessed $1^{\text {st }}$ April 2014].

11. Procopiou, M., Finney, H., Akker, S. A., Chew, S. L., Drake, W. M., Burrin, J. 2009, "Evaluation of an enzyme immunoassay for plasma-free metanephrines in the diagnosis of catecholamine-secreting tumors", European Journal of Endocrinology, no. 161, pp. 131-140.

12. Pheochromocytoma, 2014, 2014, [online]. Available from: http://www.nlm.nih.gov/medlineplus/pheoc hromocytoma.html [Accessed 25 March 2014].

13. Sanath, K., Rohit, D., Nicholas, W., ElShafie, O., \& Karin, N. 2014, "Successful Management of Pheochromocytoma using Preoperative Oral Labetalol and Intraoperative Management Sulphate", Sultan Qaboos University Medical Journal, vol. 14 , no. 2, pp. 236-240.

14. Scottish Intercollegiate Guidelines Network (SIGN), 2014, [online]. Available from: http://www.sign.ac.uk/methodology/checkli sts.html [Accessed 25 March 2014].

15. Vaclavik, J., Stejskal, D., Lacnak, B., Lazarova, M., Jedelsky, L., Kadalova, L., et al. 2007, "Free plasma metanephrines as a screening test for pheochromocytoma in low-risk patients", Journal of Hypertension, vol. 25, no. 7, pp. 1427-1431.

16. Yogish, C., Kudva, Anna, M., Sawka, William, F., \& Young, J. R., 2003, "The Laboratory Diagnosis of Adrenal Pheochromocytoma: The Mayo Clinic Experience", The Journal of Clinical Endocrinology \& metabolism, vol. 88, no. 10, pp. 4533-4539. 